\title{
Editorial: Emerging Technologies for Sustainable Development: From Smart Cities to Circular Economy
}

\author{
Lip Huat Saw ${ }^{1 *}$, Agus P. Sasmito ${ }^{2}$ and Jin Xuan ${ }^{3}$ \\ ${ }^{1}$ Lee Kong Chian Faculty of Engineering and Science, UTAR, Kajang, Malaysia, ${ }^{2}$ Department of Mining and Materials Engineering, \\ McGill University, Montreal, QR, Canada, ${ }^{3}$ Department of Chemical Engineering, Loughborough University, Loughborough, \\ United Kingdom
}

Keywords: complex urban energy system, syngas production, solar desalination, energy business, solar energy, wind energy, microgrid policy, battery

\section{Editorial on the Research Topic}

Emerging technologies for sustainable development: From smart cities to circular economy

It is projected that about $60 \%$ of the world's population is expected to live in cities by 2050 and investment in smart city technology is expected to grow to $\$ 135$ billion by 2021 . Nowadays, major metropolitan areas consume around $65 \%$ of available primary energy and contribute around $70 \%$ of greenhouse gas emissions for heating and cooling, illumination and transport. In addition, over 720 billion tons of waste is produced every year in worldwide cities. Hence, innovative solutions for the energy-environment are needed to develop sustainable cities. These include smart and carbon-free transport, the use of renewable energy, effective greenhouse gas emissions management to reduce carbon footprint and pollutants to improve air and soil quality as well as waste management through and recycling by transforming conventional linear economy to circular economy.

The research topic on the emerging technologies for sustainable development: From smart cities to circular economy consists of seven articles from various authors which offer solutions for renewable energy and clean water. The article research topic span from investment planning for complex urban energy systems, syngas production using palm kernel shells, solar desalination with Fresnel lens, energy business in Thailand, impact of El Nino-southern oscillation to the wind and solar data in Malaysia, microgrid policies in Thailand and aluminium-air battery.

Mixed-integer linear programming formulations using time slices is commonly used for the design and optimization of urban multi-energy systems. Bornand et al. used mixed-integer linear programming to investigate the various energy supply and demand, heating and cooling requirements and resource availability for the hospital. Space heating, cooling and ventilation account for almost one-third of the energy consumption for hospitals in central Europe. The investigation results indicate that priority should be given to retrofit the building with a large aspect ratio to reduce the thermal load and supply temperature of the hydronic network. This is important to reduce the energy consumption in the hospital.

Dechapanya et al. used biochar produced from the pyrolysis of mangosteen and durian peels as tar removal and $\mathrm{NiO} / \mathrm{CaO}$-based catalysts in the palm kernel shells syngas production. The results show that increasing the $\mathrm{NiO}$ amounts in the catalyst will increase the production of methane and carbon dioxide concentrations in the syngas. On the other hand, incorporation of the biochar in the syngas production will lead to an increase of the catalytic unit to about 9 times compared to syngas production without biochar.

Choong et al. investigated the production of potable water from saline water through the solar desalination process. Flat radial Fresnel lenses were used in the study due to its high achievable temperature and focal point. Compared to a single Fresnel lens, multiple Fresnel lenses will offer 
higher potable water production rate. Besides, length/width ratio of the solar still setup is also another important factor affect the desalination performance.

Nakapreecha et al. analysed the Thai energy business in the next 30 years by looking at different aspects such as social, technological, economical, ecological and political. The authors found that policy and technology are the main factors driving the Thailand energy business in the next 30 years. Moreover, the authors also highlighted that the allocation of the investment for the energy transition to achieve the sustainable development goals remains an unanswered question and needs further investigation.

Albani et al. applied wavelet analysis to investigate the impact of El-Nino southern oscillation events on the solar irradiation and wind speed in Malaysia. The analysis shows that the solar irradiation will increase due to the El-Nino and decrease due to the La-Nina period. On the other hand, the wind speed shows a reverse trend. The wind speed will increase during the La-Nina period and decrease during the El-Nino period. In addition, Northeast monsoon season will cause high wind speed at the east coast of peninsular Malaysia, south-western of Sarawak, northern and north-eastern of Sabah.

Meenual and Usapein reviewed the microgrid policy in Thailand. There are various types of renewable energy resources used in Thailand such as solar, wind, biomass and mini hydro. Currently there are four types of microgrid in Thailand such as campus microgrid, utility microgrids, business microgrids and foreign-funded microgrids. Majority of the microgrids are driven by public policy and legal flexibility. The key drivers for the Thailand microgrid policy are electricity access, wealth creation and distribution, environmental protection, and technology development. The study also shows that advancement of peer-to-peer trading and blockchain are the main driving forces for microgrids in urban areas. On the other hand, the main driving force for microgrids in rural areas will be reliable and resilient power supply.

Tan et al. developed the polypropylene-based aluminium-air battery. The anode of the battery is made of aluminium foil, carbon fibre cloth as the air-cathode and polypropylene and kimwipes as the battery separator. The electrolyte used in the aluminium-air battery is potassium hydroxide. The performance of the polypropylene-based aluminium-air battery is benchmarked with paper-based aluminium-air battery for different discharged currents and different concentrations of electrolyte. It is found that the performance of the aluminium-air battery with polypropylene separator is about 3 times better than the aluminium-air battery with paper-based separator for different discharge currents using $1 \mathrm{Mol}$ of potassium hydroxide electrolyte.

In summary, this research topic highlights the different areas of interest to the energy community including optimization of energy usage in hospitals, syngas production, solar desalination, energy business and microgrid policy, effect of El-Nino and LaNina on the wind energy harvesting and aluminium-air battery. As energy is one of the important elements in the 17 United Nations' Sustainable Development Goals (SDGs) to combat the climate change and poverty eradication, we foresee more research work and publication from the worldwide researchers Lastly, we would like to thank all the contributing authors, reviewers, journal editors and publication team for their invaluable support for this research topics.

\section{AUTHOR CONTRIBUTIONS}

$\mathrm{SH}$, leading guest editor, AS, guest editor and JX, guest editor. All authors listed have made a substantial, direct and intellectual contribution to the work, and approved it for publication.

\section{FUNDING}

This work was supported by Fundamental Research Grant Scheme (Grant No. FRGS/1/2018/TK07/UTAR/02/4) from the Ministry of Higher Education Malaysia.

Conflict of Interest: The authors declare that the research was conducted in the absence of any commercial or financial relationships that could be construed as a potential conflict of interest.

Publisher's Note: All claims expressed in this article are solely those of the authors and do not necessarily represent those of their affiliated organizations, or those of the publisher, the editors and the reviewers. Any product that may be evaluated in this article, or claim that may be made by its manufacturer, is not guaranteed or endorsed by the publisher.

Copyright $\odot 2021$ Saw, Sasmito and Xuan. This is an open-access article distributed under the terms of the Creative Commons Attribution License (CC BY). The use, distribution or reproduction in other forums is permitted, provided the original author(s) and the copyright owner(s) are credited and that the original publication in this journal is cited, in accordance with accepted academic practice. No use, distribution or reproduction is permitted which does not comply with these terms. 